\title{
Flows Associated with Ergodic Non-Singular Transformation Groups
}

\author{
By \\ Toshihiro Hamachi, ${ }^{*}$ Yukimasa OKA** and Motosige OsIKawa*
}

\section{§0. Introduction}

In this paper we shall give a nice invariant for the weak equivalence of ergodic non-singular transformation groups. It is a one-parameter ergodic non-singular flow associated with an ergodic non-singular transformation group. Since in 1960 an example of an ergodic non-singular transformation without $\sigma$-finite invariant measures was given in Ergodic theory [16], the structure and the classification of ergodic non-singular transformations have been studied by many authors ([2], [4], [5], [6], [7], [8] [11] and [13]). Among these works, Krieger's weak equivalence theory is fundamental in the classification problem of ergodic nonsingular transformation groups without $\sigma$-finite invariant measures. This classification is closely connected with the classification of type III factors in the theory of von Neumann algebras ([15]).

The Tomita-Takesaki theory of generalized Hilbert algebras ([18]) plays important roles in the analysis of type III factors. Using this theory, A. Connes [3] introduced algebraic invariants - the S-set $\mathrm{S}(M)$ and the $\mathrm{T}$-set $\mathrm{T}(M)$ - for a factor $M$ and obtained a classification of type III factors. M. Takesaki [19] introduced the dual action of the modular automorphism group and obtained the structure theorem of type III factors. In the classification problem of ergodic non-singular transformation groups $G, W$. Krieger [10] and the present authors [6] introduced invariants $\mathrm{r}(G)$ and $\mathrm{T}(G)$ respectively, both of which are

Communicated by H. Araki, August 5, 1974.

* Department of Mathematics, College of General Education, Kyushu University, Fukuoka.

** Department of Mathematics, Faculty of Science, Kyushu University, Fukuoka. 
closely related to the existence problem of $\sigma$-finite invariant measures. The invariant $\mathrm{r}(G)$ and $\mathrm{T}(G)$ are nothing but the S-set and T-set of the group measure space construction factor $M_{G}$ of $G$. They are also corresponding to the Araki-Woods invariant $\mathrm{r}_{\infty}\left(M_{G}\right)$ and $\rho\left(M_{G}\right)$ ([1]) of the factor $M_{G}$ in the case of an infinite product type transformation group $G$.

By the metrical properties of the associated flows, we can obtain much more informations about non-singular transformation groups. The T-set is the point spectrum of the associated flow and the S-set is illustrated by only the periodic motion of the associated flow. It is shown by Krieger's skew-product method ([9]) that any ergodic measurable measure preserving flow is realized as the associated flow of an ergodic non-singular transformation group. We study the weakly equivalent classes of the product $G \times G^{\prime}$ of ergodic non-singular transformation groups by using its associated flow and we introduce a new class (type III $^{\Gamma}$ ) of non-singular transformation groups of type III. Also we can obtain examples of ergodic non-singular transformation groups $G$ of type III satisfying that $G \times G$ is not weakly equivalent with $G$. We show the Araki-Woods characterization theorem of the asymptotic ratio set and the $\rho$-set in the sense of weak equivalence of non-singular transformation groups. By a characterization of a dissipative non-singular flow, we give another proof of the existence of an invariant measure under the condition $\mathrm{T}(G)=\mathbf{R}([6])$.

Professor H. Araki showed us that Takesaki's dual action ([19]) of the modular automorphism group of the group measure space construction factor is realized by the associated flow. Professor W. Krieger informed us that he introduced a non-singular flow for an ergodic nonsingular transformation and proved the one-to-one correspondence between the weak equivalence of ergodic non-singular transformations and the strong equivalence of flows $([12])$.

\section{§1. Preliminaries}

Let $(\Omega, \mathfrak{F}, P)$ be a Lebesgue measure space. Two measures $\mu$ and $v$ on the measurable space $(\Omega, \mathfrak{F})$ are equivalent $\mu \sim v$, when $\mu(A)=0$ if and only if $v(A)=0, A \in \mathfrak{F}$. A 1-1 mapping $g$ from $\Omega$ onto itself is 
a non-singular transformation if it is bi-measurable (i.e. $g^{-1} \mathfrak{F} \subset \mathfrak{F}$ and $g \mathfrak{F} \subset \mathfrak{F})$ and $P g \sim P$ where $P g(A)=P(g A), A \in \mathfrak{F}$. Let $G$ be a countable group of non-singular transformations of $(\Omega, \mathfrak{F}, P)$. A measure $\mu$ defined on $(\Omega, \mathfrak{F})$ is $G$-invariant if $\mu g=\mu, g \in G$ and a measurable function $f(\omega)$ is $G$-invariant if $f(g \omega)=f(\omega), g \in G$, a.e. $\omega$. $G$ is ergodic if every $G$-invariant function on $(\Omega, \mathfrak{F}, P)$ is a constant a.e. We denote by $[G]$ the group of all non-singular transformations $g$ of $(\Omega, \mathbb{F}, P)$ satisfying that there exist measurable sets $A_{n} \in \mathfrak{F}, n=1,2, \ldots$ and nonsingular transformations $g_{n} \in G, n=1,2, \ldots$ such that $\Omega=\bigcup_{n=1}^{\infty} A_{n}$ (disjoint) and $g \omega=g_{n} \omega$, a.e. $\omega \in A_{n}, n=1,2, \ldots$. The group $[G]$ is said to be the full group of $G$. Two countable non-singular transformation groups $G$ and $G^{\prime}$ of $(\Omega, \mathfrak{F}, P)$ and $\left(\Omega^{\prime}, \mathfrak{F}^{\prime}, P^{\prime}\right)$ respectively, are called weakly equivalent if there exists a bi-measurable 1-1 mapping $\varphi$ from $\Omega$ onto $\Omega^{\prime}$ such that $\varphi[G] \varphi^{-1}=\left[G^{\prime}\right]$ and $P \sim P^{\prime} \varphi$.

Let us now define the ratio set $\mathrm{r}(G)$ and the $\mathrm{T}$-set $\mathrm{T}(G)$ of a countable non-singular transformation group $G$ of $(\Omega, F, P)$. The ratio set $\mathrm{r}(G)$ is the closure of the set of all positive numbers $r$ satisfying that for any $\varepsilon>0$ and any measurable set $A$ with $P(A)>0$ there exists a measurable subset $B$ of $A$ with $P(B)>0$ and $g \in G$ such that $g B \subset A$ and $r e^{-\varepsilon}<\frac{\mathrm{d} P g}{\mathrm{~d} P}(\omega)<r e^{\varepsilon}, \omega \in B([10])$, and the $\mathrm{T}$-set $\mathrm{T}(G)$ is the set of all real numbers $t$ satisfying that there exists a measurable function $\exp i \xi(\omega)$ such that $\exp i\{\xi(g \omega)-\xi(\omega)\}=\exp i t \log \frac{\mathrm{d} P g}{\mathrm{~d} P}(\omega), g \in G$, a.e. $\omega([6])$. The set $\mathrm{r}(G) \backslash\{0\}$ is a multiplicative subgroup of positive numbers and $\mathrm{T}(G)$ is an additive subgroup of $\mathbf{R}$. These two sets are invariants for the weak equivalence.

For a countable ergodic non-singular transformation group $G$ of $(\Omega$, $\mathfrak{F}, P)$ a pair $(\mu, H)$ is said to be an admissible pair of $G$ if $\mu$ is a $\sigma$-finite measure equivalent with $P$ and if $H$ is an ergodic subgroup consisting of $\mu$-preserving transformations of $[G]$. The set $\Delta(\mu, H)$ $=\left\{r>0 \mid\right.$ for any $\varepsilon>0$ there exists $g \in[G]$ such that $P\left(r e^{-\varepsilon}<\frac{\mathrm{d} \mu g}{\mathrm{~d} \mu}(\omega)\right.$ $\left.\left\langle r e^{\varepsilon}\right)>0\right\}$ is a closed multiplicative subgroup of $\mathbf{R}_{+}$if $(\mu, H)$ is an admissible pair of $G$. This set is independent of the choice of an admissible pair ([6]).

For a countable ergodic non-singular transformation group $G$ of ( $\Omega$, $\mathfrak{F}, P$ ) we consider the following cases: 
(a) There exists an equivalent $\sigma$-finite invariant measure $\mu$, or equivalently $(\mu, G)$ is an admissible pair of $G$.

$\left(\mathrm{b}_{\lambda}\right)$ There exist an admissible pair $(\mu, H)$ and $0<\lambda<1$ such that $\Delta(\mu, H)=\left\{\lambda^{n} \mid-\infty<n<\infty\right\}$.

(c) There exists an admissible pair $(\mu, H)$ such that $\Delta(\mu, H)=(0$, $\infty)$

(d) There is no admissible pair.

These cases are exclusive and exaustive.

Definition ([6]). Let $G$ be a countable ergodic non-singular transformation group of $(\Omega, \mathfrak{F}, P)$. (1) We say that $G$ is of type III if $G$ has no equivalent $\sigma$-finite invariant measures. (2) We say that $G$ is of type $\mathrm{III}_{\lambda}, 0<\lambda<1, \mathrm{III}_{1}$ or $\mathrm{III}_{0}$ accordingly as the case $\left(\mathrm{b}_{\lambda}\right),(\mathrm{c})$ or (d) happens.

Note that the type of $G$ is an invariant under the weakly equivalent relation and that if $G$ is of type III $_{1}$ then for any null set $N \in \mathfrak{F}$ the set $\left\{\log \frac{\mathrm{d} \mu g}{\mathrm{~d} \mu}(\omega) \mid g \in G, \omega \notin N\right\}$ contains at least two rationally independent real numbers.

\section{§2. The Associated Flow $\left\{\widetilde{T}_{s}\right\}_{-\infty<s<+\infty}$}

Definition 1. A one-parameter group $\left\{U_{s}\right\}_{-\infty<s<+\infty}$ of non-singular transformations of $\left(X, \mathfrak{B}_{X}, \mu_{X}\right)$, which we call simply a non-singular flow, is measurable if the mapping $\mathbf{R} \times X \ni(s, x) \rightarrow U_{s} x \in X$ is measurable.

Let $G$ be a countable non-singular transformation group acting on a Lebesgue measure space $(\Omega, \mathfrak{F}, P)$. We denote by $\tilde{G}$ the group of following non-singular transformations $\tilde{g}$ on $(\Omega \times \mathbf{R}, \mathfrak{F} \times \mathfrak{B}(\mathbf{R}), \mathrm{d} P \times \mathrm{d} u)$;

$$
\tilde{g}(\omega, u)=\left(g \omega, u+\log \frac{\mathrm{d} P g}{\mathrm{~d} P}(\omega)\right), \quad g \in G
$$

Let $\zeta(\widetilde{G})$ be the measurable partition $([17])$ generated by all $\widetilde{G}$-invariant measurable sets. For $-\infty<s<+\infty$, put $T_{s}(\omega, u)=(\omega, u+s),(\omega, u) \in \Omega$ $\times \mathbf{R}$. Since $\left\{T_{s}\right\}_{-\infty<s<+\infty}$ commutes with $\tilde{G}$, we can define the factor flow $\left\{\widetilde{T}_{s}\right\}_{-\infty<s<+\infty}$ of $\left\{T_{s}\right\}_{-\infty<s<+\infty}$ on the quotient space $\Omega \times \mathbf{R} / \zeta(\widetilde{G})$. 
$\widetilde{T}_{s}(-\infty<s<+\infty)$ is a non-singular transformation with respect to any $\sigma$-finite measure equivalent with the image measure of $\mathrm{d} P \times \mathrm{d} u$ and $\left\{\widetilde{T}_{s}\right\}_{-\infty<s<+\infty}$ is a measurable flow.

Definition 2. We call the factor flow $\left\{\widetilde{T}_{s}\right\}_{-\infty<s<+\infty}$ the non-singular flow associated with the non-singular transformation group $G$ or simply the associated flow of $G$.

We note that the associated flow $\left\{\widetilde{T}_{s}\right\}_{-\infty<s<+\infty}$ of $G$ is ergodic if and only if $G$ is ergodic.

Definition 3. Non-singular flows $\left(X, \mathfrak{B}_{X}, \mu_{X} ;\left\{U_{s}\right\}_{-\infty<s<+\infty}\right)$ and ( $Y$, $\left.\mathfrak{B}_{Y}, \mu_{Y} ;\left\{V_{s}\right\}_{-\infty<s<+\infty}\right)$ are mutually strongly equivalent if there exists a bi-measurable 1-1 mapping $\psi$ from $X$ onto $Y$ such that $\mu_{X} \sim \mu_{Y} \psi$ and for all $-\infty<s<+\infty, \psi U_{s} x=V_{s} \psi x$, a.e. $x$.

We note that the strong equivalence among ergodic non-singular flows is the same as the metrically isomorphic equivalence if they admit finite equivalent invariant measures.

Theorem 1. If ergodic non-singular transformation groups ( $\Omega$, $\mathfrak{F}, P ; G)$ and $\left(\Omega^{\prime}, \mathfrak{F}^{\prime}, P^{\prime} ; G^{\prime}\right)$ are mutually weakly equivalent, then their associated flows are mutually strongly equivalent.

Proof. Let $\varphi$ be a bi-measurable 1-1 mapping from $\Omega$ onto $\Omega^{\prime}$ such that $\varphi[G] \varphi^{-1}=\left[G^{\prime}\right]$ and $P \sim P^{\prime} \varphi$. Put $\psi(\omega, u)=\left(\varphi \omega, u+\log \frac{\mathrm{d} P^{\prime} \varphi}{\mathrm{d} P}(\omega)\right)$. Then $\psi$ is a $1-1$ mapping from $\Omega \times \mathbf{R}$ onto $\Omega^{\prime} \times \mathbf{R}$ and satisfies that for $-\infty<s<+\infty, \psi T_{s}(\omega, u)=T_{s}^{\prime} \psi(\omega, u)$, a.e. $(\omega, u) \in \Omega \times \mathbf{R}$. It is enough to show that $f(\psi(\omega, u))$ is a $\widetilde{G}$-invariant measurable function for any $\tilde{G}^{\prime}$ invariant measurable function $f\left(\omega^{\prime}, u\right)$. For $g \in[G]$ and $g^{\prime}=\varphi g \varphi^{-1}$ $\in\left[G^{\prime}\right]$,

$$
\begin{aligned}
f\left(\psi\left(g \omega, u+\log \frac{\mathrm{d} P g}{\mathrm{~d} P}(\omega)\right)\right) & =f\left(\varphi g \omega, u+\log \frac{\mathrm{d} P g}{\mathrm{~d} P}(\omega)+\log \frac{\mathrm{d} P^{\prime} \varphi}{\mathrm{d} P}(g \omega)\right) \\
& =f\left(g^{\prime} \varphi \omega, u+\log \frac{\mathrm{d} P g}{\mathrm{~d} P}(\omega)+\log \frac{\mathrm{d} P^{\prime} g^{\prime} \varphi}{\mathrm{d} P g}(\omega)\right) \\
& =f\left(g^{\prime} \varphi \omega, u+\log \frac{\mathrm{d} P^{\prime} g^{\prime}}{\mathrm{d} P^{\prime}}(\varphi \omega)+\log \frac{\mathrm{d} P^{\prime} \varphi}{\mathrm{d} P}(\omega)\right)
\end{aligned}
$$




$$
\begin{aligned}
& =f\left(\tilde{g}^{\prime}\left(\varphi \omega, u+\log \frac{\mathrm{d} P^{\prime} \varphi}{\mathrm{d} P}(\omega)\right)\right) \\
& =f(\psi(\omega, u)) .
\end{aligned}
$$

Definition 4. Let $\left(X, \mathfrak{B}_{X}, \mu_{X} ;\left\{U_{s}\right\}_{-\infty<s<+\infty}\right)$ be a non-singular flow. A real number $t$ belongs to the set $\sigma\left(\left\{U_{s}\right\}\right)$, which is called the point spectrum of $\left\{U_{s}\right\}_{-\infty<s<+\infty}$, if there exists a measurable function $\exp i \xi(x)$ such that for all $-\infty<s<+\infty$

$$
\exp i \xi\left(U_{s} x\right)=\exp i t s \cdot \exp i \xi(x), \quad \text { a.e. } x \text {. }
$$

Theorem 2. Let $G$ be a countable non-singular transformation group and $\left\{\widetilde{T}_{s}\right\}_{-\infty<s<+\infty}$ be its associated flow. Then the T-set is $\mathrm{T}(G)=\sigma\left(\left\{\tilde{T}_{s}\right\}\right)$.

Proof. Let $t \in \mathrm{T}(G)$. Then

$$
\exp i\{\xi(g \omega)-\xi(\omega)\}=\exp i t \log \frac{\mathrm{d} P g}{\mathrm{~d} P}(\omega), \quad g \in G, \quad \text { a.e. } \omega
$$

for some measurable function $\exp i \xi(\omega)$. If we put $f(\omega, u)=\exp i\{t u$ $-\xi(\omega)\}$, then $f(\omega, u+s)=\exp i t s \cdot f(\omega, u)$ and

$$
\begin{aligned}
f\left(g \omega, u+\log \frac{\mathrm{d} P g}{\mathrm{~d} P}(\omega)\right) & =\exp \{-i \xi(g \omega)\} \exp i t\left(u+\log \frac{\mathrm{d} P g}{\mathrm{~d} P}(\omega)\right) \\
& =\exp i t u \cdot \exp \{-i \xi(\omega)\} \\
& =f(\omega, u), \quad g \in G, \text { a.e. }(\omega, u) .
\end{aligned}
$$

Conversely, let $t \in \sigma\left(\left\{\widetilde{T}_{s}\right\}\right)$. Then

$$
\exp i \eta(\omega, u+s)=\exp i t s \cdot \exp i \eta(\omega, u), \quad-\infty<s<+\infty,
$$

for some $\tilde{G}$-invariant measurable function $\exp i \eta(\omega, u)$. If we put $\exp \{-i \xi(\omega)\}=\exp i \eta(\omega, 0)$, then

$$
\exp i\{\xi(g \omega)-\xi(\omega)\}=\exp i t \log \frac{\mathrm{d} P g}{\mathrm{~d} P}(\omega), \quad g \in G, \quad \text { a.e. } \omega . \quad \text { Q.E.D. }
$$

Theorem 3. Let $\left\{\widetilde{T}_{s}\right\}_{-\infty<s<+\infty}$ be the non-singular flow associ- 
ated with an ergodic countable non-singular transformation group $G$ of $(\Omega, \mathfrak{F}, P)$. Then

(1) $G$ admits an equivalent $\sigma$-finite invariant measure if and only if $\left\{\tilde{T}_{s}\right\}_{-\infty<s<+\infty}$ is strongly equivalent with the translation

$$
\mathbf{R} \ni u \rightarrow u+s, \quad-\infty<s<+\infty,
$$

(2) $G$ is of type $I I I_{\lambda}(0<\lambda<1)$ if and only if $\left\{\tilde{T}_{s}\right\}_{-\infty<s<+\infty}$ is strongly equivalent with the periodic flow $[0,-\log \lambda) \ni u \rightarrow u+s(\bmod ,-\log \lambda)$, $-\infty<s<+\infty$,

(3) $G$ is of type $I I I_{1}$ if $\left\{\widetilde{T}_{s}\right\}_{-\infty<s<+\infty}$ is the trivial flow, and the converse is true under the assumption that $G$ is a cyclic group, and (4) $G$ is of type $I I I_{0}$ if and only if $\left\{\tilde{T}_{s}\right\}_{-\infty<s<+\infty}$ is an ergodic, aperiodic and conservative flow, under the assumption that $G$ is a cyclic group.

Proof. (1) Let $\mu$ be an equivalent $\sigma$-finite $G$-invariant measure. Then the measurable partition $\zeta(\widetilde{G})$ is equal to $\{\Omega \times\{u\} ; u \in \mathbf{R}\}$ since $\widetilde{G}=\left\{\tilde{g} ; \tilde{g}(\omega, u)=\left(g \omega, u+\log \frac{\mathrm{d} \mu g}{\mathrm{~d} \mu}(\omega)\right)=(g \omega, u), g \in G\right\}$. So the factor flow of $\left\{T_{s}\right\}_{-\infty<s<+\infty}$ is the translation on $\mathbf{R}$. Conversely let $\psi$ be a bimeasurable 1-1 mapping from $\Omega \times \mathbf{R} / \zeta(\widetilde{G})$ onto $\mathbf{R}$ such that $\left\{\widetilde{T}_{s}\right\}_{-\infty<s<+\infty}$ commutes with the translation on $\mathbf{R}$ under the mapping $\psi$, and $\pi$ be the canonical mapping from $\Omega \times \mathbf{R}$ onto $\Omega \times \mathbf{R} / \zeta(\widetilde{G})$. For the measurable partition $\left\{\pi^{-1} \circ \psi^{-1}(u) ; u \in \mathbb{R}\right\}$ of $\Omega \times \mathbf{R}$ it holds that for almost all $\psi^{-1}(u)$ and for almost all $\omega, \pi^{-1} \circ \psi^{-1}(u)$ intersects $\{\omega\} \times \mathbf{R}$ in exactly one point $(\omega, u-\psi(\pi(\omega, 0)))$ from the assumption. Denote for a fixed cross section $\pi^{-1} \circ \psi^{-1}(u), \mathrm{d} \mu(\omega)=\exp \{-u+\psi(\pi(\omega, 0))\} \mathrm{d} P(\omega)$. Notice that a $\omega$ function $-u+\psi(\pi(\omega, 0))$ is measurable. Then the equivalent measure $\mu$ is $G$-invariant. Indeed, since $\tilde{g}(\omega, u-\psi(\pi(\omega, 0)))=(g \omega, u-\psi(\pi(\omega, 0))$ $\left.+\log \frac{\mathrm{d} P g}{\mathrm{~d} P}(\omega)\right) \in \pi^{-1} \circ \psi^{-1}(u)$, we have $\psi(\pi(g \omega, 0))=\psi(\pi(\omega, 0))-\log \frac{\mathrm{d} P g}{\mathrm{~d} P}(\omega)$, a.e. $\omega, g \in G$. Therefore

$$
\begin{aligned}
\mathrm{d} \mu(g \omega) & =\exp \{-u+\psi(\pi(g \omega, 0))\} \mathrm{d} P(g \omega) \\
& =\exp \left\{-u+\psi(\pi(\omega, 0))-\log \frac{\mathrm{d} P g}{\mathrm{~d} P}(\omega)\right\} \mathrm{d} P(g \omega)
\end{aligned}
$$




$$
\begin{aligned}
& =\exp \{-u+\psi(\pi(\omega, 0))\} \mathrm{d} P(\omega) \\
& =\mathrm{d} \mu(\omega)
\end{aligned}
$$

(2) Let $(\mu, H)$ be an admissible pair of type $\operatorname{III}_{\lambda}(0<\lambda<1)$. Then the measurable partition $\zeta(\widetilde{G})$ is $\{\underset{-\infty<n<+\infty}{\cup} \Omega \times\{u-n \log \lambda\} ; 0 \leqq u<$ $-\log \lambda\}$, since $H$ is ergodic and $\tilde{h}(\omega, u)=(h \omega, u), h \in H$ and since for almost all $\omega$ and for any integer $n$ there exists $g \in G$ such that $\log \frac{\mathrm{d} \mu g}{\mathrm{~d} \mu}(\omega)$ $=n \log \lambda$ (Section 6 of [6]). Therefore $\left\{\tilde{T}_{s}\right\}_{-\infty<s<+\infty}$ is the periodic flow $[0,-\log \lambda) \ni u \rightarrow u+s(\bmod .-\log \lambda),-\infty<s<+\infty$. Conversely let $\psi$ be a bi-measurable 1-1 mapping from $\Omega \times \mathbf{R} / \zeta(\widetilde{G})$ onto $[0,-\log \lambda)$ such that $\psi T_{s}(\omega, u)=\psi(\omega, u)+s(\bmod .-\log \lambda)$, a.e. $(\omega, u)$. Then for almost all $\psi^{-1}(u)$ and for almost all $\omega, \pi^{-1} \circ \psi^{-1}(u)$ intersects each set $\{\omega\} \times$ $[-n \log \lambda,-(n+1) \log \lambda),-\infty<n<+\infty$, in exactly one point. For a fixed $u$, the function $f(\omega)=\inf \left\{v ; v \geqq 0\right.$ and $\left.(\omega, v) \in \pi^{-1} \circ \psi^{-1}(u) \cap(\{\omega\} \times \mathbb{R})\right\}$ is measurable. We define an equivalent measure $\mathrm{d} \mu(\omega)=\exp \{-f\}(\omega) \mathrm{d} P(\omega)$. $\widetilde{G}$ acts ergodically on each ergodic component $\pi^{-1} \circ \psi^{-1}(u)$. Let $\tilde{H}$ be the induced transformation group of this action on the set $\{(\omega, f(\omega))$; $\omega \in \Omega\}$. Then $H$ is ergodic and $\mu$-preserving and $\frac{\mathrm{d} \mu g}{\mathrm{~d} \mu}(\omega) \in\left\{\lambda^{n} ;-\infty<\right.$ $n<+\infty\}, g \in G$, a.e. $\omega$.

(3) Let $(\mu, H)$ be an admissible pair of a non-singular transformation group $G$ of type III $_{1}$. If $f(\omega, u)$ is a $\widetilde{G}$-invariant measurable function, then for almost all $(\omega, u), f(h \omega, u)=f(\omega, u), h \in H$. From the ergodicity of $H, f(\omega, u)=f(u)$, a.e. $(\omega, u)$ and so for almost all $(\omega, u), f\left(u+\log \frac{\mathrm{d} \mu g}{\mathrm{~d} \mu}(\omega)\right)$ $=f(u), g \in G$. Let $\Gamma=\{t \in \mathbf{R} ; f(u+t)=f(u)$, a.e. $u\}$ then $\Gamma$ is a subgroup of $\mathbf{R}$ and contains at least two rationally independent real numbers by the definition of type $\mathrm{III}_{1}$. Thus $\Gamma$ is a dense subgroup. Therefore $f(u)=$ constant, a.e.u. Conversely let $G$ be a cyclic group and let $G$ be ergodic. Then for any set $A \in \mathfrak{F}$ with positive measure, any positive number $r$ and any positive number $\varepsilon$, there exist a measurable subset $\widetilde{B} \subset A \times[-\varepsilon, \varepsilon]$ with positive measure and $g \in G$ such that $\tilde{g} \widetilde{B}$ $\subset A \times[\log r-\varepsilon, \log r+\varepsilon]$. Put $B=\{\omega \in A ;(\omega, u) \in \widetilde{B}$ for some $u\}$. Then $P(B)>0, g B \subset A$ and $r \exp \{-2 \varepsilon\}<\frac{\mathrm{d} P g}{\mathrm{~d} P}(\omega)<r \exp 2 \varepsilon, \omega \in B$. Therefore the ratio set is $r(G)=[0,+\infty)$. By Theorem 2.8 of $[10], G$ is of type III $_{1}$. 
(4) Since an ergodic and dissipative non-singular flow on a Lebesgue space is metrically isomorphic with the translation on $\mathbb{R}$, (4) follows from (1), (2) and (3) in the case that $G$ is a cyclic group. Q.E.D.

Problem. Is $G$ of type $\mathrm{III}_{1}$ if $\tilde{G}$ is ergodic, without the assumption that $G$ is a cyclic group?

Theorem 4. Let $(\Omega, \mathfrak{F}, P ; G)$ be of type $I I I_{1}$ with an admissible pair $(P, H)$ and $\left(X, \mathfrak{B}_{X}, \mu_{X} ;\left\{U_{s}\right\}_{-\infty<s<+\infty}\right)$ be a measurable ergodic measure preserving flow and let $G_{\{U\}}=\left\{g_{\{U\}} ; g \in G\right\}$ where

$$
g_{\{U\}}(\omega, x)=\left(g \omega, U_{-\log \frac{d P g}{d P}(\omega)} x\right), \quad g \in G .
$$

Then the associated flow $\left\{\widetilde{T}_{s}\right\}_{-\infty<s<+\infty}$ of the ergodic non-singular transformation group $G_{\{U\}}$ is metrically isomorphic with $\left\{U_{s}\right\}_{-\infty<s<+\infty}$.

Proof. Let $f(\omega, x, u)$ be a $\widetilde{G}_{\{U\}}$-invariant bounded measurable function. Then $f(h \omega, x, u)=f(\omega, x, u), h \in H$, a.e. $(\omega, x, u)$ and the ergodicity of $H$ implies $f(\omega, x, u)=f(x, u)$, a.e. $(\omega, x, u)$. So we have for almost all $(\omega, x, u), f\left(U_{-\log \frac{\mathrm{d} P g}{\mathrm{~d} P}(\omega)} x, u+\log \frac{\mathrm{d} P g}{\mathrm{~d} P}(\omega)\right)=f(x, u), g \in G . \quad$ Let $\Gamma=\{t \in \mathbf{R} \mid$ $f\left(U_{-t} x, u+t\right)=f(x, u)$, a.e. $\left.(x, u)\right\}$. Then $\Gamma$ is a subgroup of $\mathbf{R}$ and contains at least two rationally independent real numbers by the definition of type $\mathrm{III}_{1}$. Thus $\Gamma$ is a dense subgroup and it follows from Lemma 2 (2) of Section 4 that for any $-\infty<s<+\infty f\left(U_{-s} x, u+s\right)$ $=f(x, u)$, a.e. $(x, u)$. Conversely a $\left\{U_{-s} \times \tau_{s}\right\}_{-\infty<s<+\infty}$-invariant measurable function is $\widetilde{G}_{\{U\}}$-invariant, where $\tau_{s} u=u+s, u \in \mathbb{R},-\infty<s<+\infty$, and then $\zeta\left(\widetilde{G}_{\{U\}}\right)=\Omega \times \zeta\left(\left\{U_{-s} \times \tau_{s}\right\}\right)$. Since $\left\{U_{s}\right\}_{-\infty<s<+\infty}$ is a measurable flow, for $E \in \mathfrak{B}_{X}$ the set

$$
\left\{\left(U_{-s} x, s\right) ; x \in E,-\infty<s<+\infty\right\}=\left\{(x, s) ; U_{-s} x \in E,-\infty<s<+\infty\right\}
$$

is $\left\{U_{-s} \times \tau_{s}\right\}$-invariant and measurable in $X \times \mathbf{R}$. For a countably separating base $\left\{E_{n}\right\}_{n \geqq 1}$ of $\left(X, B_{X}, \mu_{X}\right),\left\{\pi\left(\left\{U_{-s} x, s\right) ; x \in E_{n},-\infty<s<\right.\right.$ $+\infty\})\}_{n \geqq 1}$ is a countably separating base of $X \times \mathbb{R} / \zeta\left(\left\{U_{-s} \times \tau_{s}\right\}\right)$, where $\pi$ is the canonical mapping from $X \times \mathbf{R}$ onto $X \times \mathbf{R} / \zeta\left(\left\{U_{-s} \times \tau_{s}\right\}\right)$. Therefore the measurable partition $\zeta\left(\left\{U_{-s} \times \tau_{s}\right\}\right)$ is given by $\left\{\left\{\left(U_{-r} x, r\right)\right.\right.$; $-\infty<r<+\infty\} ; x \in X\}$. We define a mapping $\psi$ from $\Omega \times X \times \mathbb{R} / \zeta\left(\widetilde{G}_{\{U\}}\right)$ 
onto $X$ by $\psi\left(\Omega \times\left\{\left(U_{-r} x, r\right) ;-\infty<r<+\infty\right\}\right)=x$. The mapping $\psi$ is bi-measurable $1-1$ and satisfies

$$
\begin{aligned}
\psi(\Omega \times & \left.\left\{\left(U_{-r} x, r+s\right) ;-\infty<r<+\infty\right\}\right) \\
& =U_{s} \psi\left(\Omega \times\left\{\left(U_{-r} x, r\right) ;-\infty<r<+\infty\right\}\right), \quad-\infty<s<+\infty .
\end{aligned}
$$

Therefore the associated flow $\left\{\tilde{T}_{s}\right\}_{-\infty<s<+\infty}$ is metrically isomorphic with $\left\{U_{s}\right\}_{-\infty<s<+\infty}$ under the mapping $\psi$ Q.E.D.

We note that, in the case that $G$ of Theorem 4 is a cyclic group of type $\mathrm{III}_{1}, G_{\{U\}}$ is of type $\mathrm{III}_{0}$ if an ergodic measure preserving flow $\left\{U_{s}\right\}_{-\infty<s<+\infty}$ is aperiodic and conservative, and that furthermore if $\left\{U_{s}\right\}_{-\infty<s<+\infty}$ is a weakly mixing flow then $\mathrm{T}\left(G_{\{U\}}\right)=\{0\}$ (cf. [3], [6]).

Let $(\Omega, \mathfrak{F}, P ; G)$ be of type $\mathrm{III}_{\lambda}, 0<\lambda<1$, and $n_{g}(\omega)$ be an integervalued function such that $\frac{\mathrm{d} P g}{\mathrm{~d} P}(\omega)=\lambda^{n_{g}(\omega)}$ and let $\left(X, \mathfrak{B}_{X}, \mu_{X} ; U\right)$ be an ergodic measure preserving transformation. W. Krieger ([11]) constructed an ergodic non-singular transformation group $G_{U}=\left\{g_{U} ; g \in G\right\}$ where $g_{U}(\omega, x)=\left(g \omega, U^{n_{g}(\omega)} x\right)$. It is easy to show that the associated flow of $G_{U}$ is metrically isomorphic with the flow built under the constant function $-\log \lambda$ with the basic transformation $U$. From this it follows that for ergodic finite measure preserving transformations $U$ and $V, G_{U}$ is weakly equivalent with $G_{V}$ if and only if $U$ is metrically isomorphic with $V([9])$.

\section{§3. The Joint Flow and the Product of Non-Singular Transformation Groups}

Let $\left\{U_{s}\right\}_{-\infty<s<+\infty}$ and $\left\{V_{s}\right\}_{-\infty<s<+\infty}$ be measurable non-singular flows acting on Lebesgue spaces $\left(X, \mathfrak{B}_{X}, \mu_{X}\right)$ and $\left(Y, \mathfrak{B}_{Y}, \mu_{Y}\right)$, respectively. Let $\left\{U_{s} \times I\right\}_{-\infty<s<+\infty}$ be a flow defined by $\left(U_{s} \times I\right)(x, y)=\left(U_{s} x, y\right)$. Since $\left\{U_{s} \times \mathrm{I}\right\}_{-\infty<s<+\infty}$ commutes with $\left\{U_{s} \times V_{-s}\right\}_{-\infty<s<+\infty}$, we can define the factor flow of the flow $\left\{U_{s} \times I\right\}_{-\infty<s<+\infty}$ on the quotient space $X \times Y \mid$ $\zeta\left(\left\{U_{s} \times V_{-s}\right\}\right)$ and denote it by $\left\{(U, V)_{s}\right\}_{-\infty<s<+\infty} \quad\left\{(U, V)_{s}\right\}_{-\infty<s<+\infty}$ is a measurable non-singular flow with respect to the image measure of $\mu_{X} \times \mu_{Y}$ on $X \times Y / \zeta\left(\left\{U_{s} \times V_{-s}\right\}\right)$. 
Definition 4. We call $\left\{(U, V)_{s}\right\}_{-\infty<s<+\infty}$ the joint flow of $\left\{U_{s}\right\}_{-\infty<s<+\infty}$ and $\left\{V_{s}\right\}_{-\infty<s<+\infty}$.

We note that $\left\{(U, V)_{s}\right\}_{-\infty<s<+\infty}$ is strongly equivalent with $\{(V$, $\left.U)_{s}\right\}_{-\infty<s<+\infty}$ and that $\sigma\left(\left\{(U, V)_{s}\right\}\right)=\sigma\left(\left\{U_{s}\right\}\right) \cap \sigma\left(\left\{V_{s}\right\}\right)$.

Theorem 5. Let $G \times G^{\prime}=\left\{g \times g^{\prime} ; g \in G, g^{\prime} \in G^{\prime}\right\}$ be the product nonsingular transformation group of countable non-singular transformation groups $G$ and $G^{\prime}$, where $g \times g^{\prime}\left(\omega, \omega^{\prime}\right)=\left(g \omega, g^{\prime} \omega^{\prime}\right), \omega \in \Omega, \omega^{\prime} \in \Omega^{\prime}$. Then the associated flow of $G \times G^{\prime}$ is strongly equivalent with the joint flow of each associated flows.

Proof. We define a mapping $\psi$ from $\Omega \times \mathbf{R} \times \Omega^{\prime} \times \mathbf{R}$ onto $\Omega \times \Omega^{\prime} \times \mathbf{R}$ as follows

$$
\psi\left(\omega, u, \omega^{\prime}, u^{\prime}\right)=\left(\omega, \omega^{\prime}, u+u^{\prime}\right)
$$

Since

$$
\tilde{g} \times \tilde{g}^{\prime}\left(\omega, u, \omega^{\prime}, u^{\prime}\right)=\left(g \omega, u+\log \frac{\mathrm{d} P g}{\mathrm{~d} P}(\omega), g^{\prime} \omega^{\prime}, u^{\prime}+\log \frac{\mathrm{d} P^{\prime} g^{\prime}}{\mathrm{d} P^{\prime}}\left(\omega^{\prime}\right)\right)
$$

and

$$
\widetilde{g \times g^{\prime}}\left(\omega, \omega^{\prime}, u\right)=\left(g \omega, g^{\prime} \omega^{\prime}, u+\log \frac{\mathrm{d} P g}{\mathrm{~d} P}(\omega)+\log \frac{\mathrm{d} P^{\prime} g^{\prime}}{\mathrm{d} P^{\prime}}\left(\omega^{\prime}\right)\right),
$$

we have

$$
\psi \cdot\left(\tilde{g} \times \tilde{g}^{\prime}\right)=\widetilde{g \times g^{\prime}} \cdot \psi, \quad g \in G, \quad g^{\prime} \in G^{\prime}
$$

Hence $\psi$ induces a mapping from the product space of the quotient spaces $(\Omega \times \mathbf{R} / \zeta(\widetilde{G})) \times\left(\Omega^{\prime} \times \mathbf{R} / \zeta\left(\widetilde{G}^{\prime}\right)\right)$ onto the quotient space $\Omega \times \Omega^{\prime} \times \mathbf{R} /$ $\zeta\left(\widetilde{G \times G^{\prime}}\right)$. Since $\psi\left(\omega, u+s, \omega^{\prime}, u^{\prime}-s\right)=\left(\omega, \omega^{\prime}, u+u^{\prime}\right)$ and $\psi\left(\omega, u+s, \omega^{\prime}\right.$, $\left.u^{\prime}\right)=\left(\omega, \omega^{\prime}, u+u^{\prime}+s\right), \psi$ induces a strongly equivalent mapping between the joint flow of the associated flows of $G$ and $G^{\prime}$ and the associated flow of $G \times G^{\prime}$.

Q.E.D.

Definition 5. For a countable additive subgroup $\Gamma$ of $\mathbf{R}$, a countable ergodic non-singular transformation group $G$ is of type III $^{T}$ if the 
associated flow $\left\{\widetilde{T}_{s}\right\}_{-\infty<s<+\infty}$ of $G$ is ergodic finite measure preserving and if it has the pure point spectrum $\Gamma$.

We note $\mathrm{III}^{\frac{2 \pi}{\log \lambda} \mathrm{Z}}=\mathrm{III}_{\lambda}$, and $\mathrm{III}^{\{0\}}=\mathrm{III}_{1}$ (in case of cyclic group) by Theorem 3 and von Neumann's theorem that ergodic finite measure preserving flows with the same pure point spectrum are mutually metrically isomorphic ([14]).

Theorem 6. (1) Let $G$ be of type $I I I^{\Gamma}$ and $G^{\prime}$ be any countable ergodic non-singular transformation group. Then $G \times G^{\prime}$ is of type $I I I^{\Gamma \cap T\left(G^{\prime}\right)}$.

(2) Let $G$ and $G^{\prime}$ be countable ergodic non-singular transformation groups whose associated flows have finite invariant measures. Then $G \times G^{\prime}$ is of type $I I I^{T(G) \cap T\left(G^{\prime}\right)}$.

Proof. The proof follows from the next lemma.

Lemma 1. (1) Let $\left(X, \mathfrak{B}_{X}, \mu_{X} ;\left\{U_{s}\right\}_{-\infty<s<+\infty}\right)$ be an ergodic finite measure preserving flow which has the pure point spectrum and ( $Y$, $\left.\mathfrak{B}_{Y}, \mu_{Y} ;\left\{V_{s}\right\}_{-\infty<s<+\infty}\right)$ be an ergodic non-singular flow. Then the joint flow $\left\{(U, V)_{s}\right\}_{-\infty<s<+\infty}$ is ergodic finite measure preserving and has the pure point spectrum $\sigma\left(\left\{U_{s}\right\}\right) \cap \sigma\left(\left\{V_{s}\right\}\right)$.

(2) Let $\left(X, \mathfrak{B}_{X}, \mu_{X} ;\left\{U_{s}\right\}_{-\infty<s<+\infty}\right)$ and $\left(Y, \mathfrak{B}_{Y}, \mu_{Y} ;\left\{V_{s}\right\}_{-\infty<s<+\infty}\right)$ be ergodic finite measure preserving flows. Then the joint flow $\{(U$, $\left.V)_{s}\right\}_{-\infty<s<+\infty}$ is ergodic finite measure preserving and has the pure point spectrum $\sigma\left(\left\{U_{s}\right\}\right) \cap \sigma\left(\left\{V_{s}\right\}\right)$.

Proof. If $\mu_{X}$ is a $\left\{U_{s}\right\}_{-\infty<s<+\infty}$-invariant finite measure and if $\mu_{Y}$ is a finite measure, $\left\{(U, V)_{s}\right\}_{-\infty<s<+\infty}$ preserves the image measure of $\mu_{X} \times \mu_{Y}$ on the quotient space $X \times Y / \zeta\left(\left\{U_{s} \times V_{-s}\right\}\right)$. There exist measurable functions $\exp i \xi_{t}(x)$ for $t \in \sigma\left(\left\{U_{s}\right\}\right)$ such that $\exp i \xi_{t}\left(U_{s} x\right)=\exp i t s$. $\exp i \xi_{t}(x)$ and measurable functions $\exp i \eta_{t}(y)$ for $t \in \sigma\left(\left\{V_{s}\right\}\right)$ such that $\exp i \eta_{t}\left(V_{s} y\right)=\exp i t s \cdot \exp i \eta_{t}(y)$. Since

$$
\begin{aligned}
\exp i \xi_{t}\left(U_{s} x\right) \exp i \eta_{t}\left(V_{-s} y\right) & =\exp i t s \cdot \exp i \xi_{t}(x) \times \exp \{-i t s\} \exp i \eta_{t}(y) \\
& =\exp i \xi_{t}(x) \exp i \eta_{t}(y)
\end{aligned}
$$


for $t \in \sigma\left(\left\{U_{s}\right\}\right) \cap \sigma\left(\left\{V_{s}\right\}\right)$, exp $i \xi_{t}(x) \exp i \eta_{t}(y)$ is a $\left\{U_{s} \times V_{-s}\right\}_{-\infty<s<+\infty}$-invariant function.

We will show that the set of all $\left\{U_{s} \times V_{-s}\right\}_{-\infty<s<+\infty}$-invariant square integrable functions are generated by $\left\{\exp i \xi_{t}(x) \exp i \eta_{t}(x) ; t \in \sigma\left(\left\{U_{s}\right\}\right)\right.$ $\left.\cap \sigma\left(\left\{V_{s}\right\}\right)\right\}$. Let $f(x, y)$ be a bounded $\left\{U_{s} \times V_{-s}\right\}_{-\infty<s<+\infty}$-invariant measurable function and assume

$$
\left\langle f(\cdot, \cdot), \exp i \xi_{t}(\cdot) \exp i \eta_{t}(\cdot)\right\rangle_{L^{2}\left(\mu_{X} \times \mu_{Y}\right)}=0
$$

for $t \in \sigma\left(\left\{U_{s}\right\}\right) \cap \sigma\left(\left\{V_{s}\right\}\right)$. Define

$$
\hat{f}_{t}(y)=\left\langle f(\cdot, y), \exp i \xi_{t}(\cdot)\right\rangle_{L^{2}\left(\mu_{X}\right)}, \quad y \in Y,
$$

for $t \in \sigma\left(\left\{U_{s}\right\}\right)$. Then we have

$$
\begin{aligned}
\hat{f}_{t}\left(V_{s} y\right) & =\left\langle f\left(\cdot, V_{s} y\right), \exp i \xi_{t}(\cdot)\right\rangle_{L^{2}\left(\mu_{X}\right)} \\
& =\left\langle f\left(U_{s}, y\right), \exp i \xi_{t}(\cdot)\right\rangle_{L^{2}\left(\mu_{X}\right)} \\
& =\left\langle f(\cdot, y), \exp i \xi_{t}\left(U_{-s^{-}}\right)\right\rangle_{L^{2}\left(\mu_{X}\right)} \\
& =\left\langle f(\cdot, y), \exp \{-i t s\} \cdot \exp i \xi_{t}(\cdot)\right\rangle_{L^{2}\left(\mu_{X}\right)} \\
& =\exp \{i t s\} \hat{f}_{t}(y), \quad t \in \sigma\left(\left\{U_{s}\right\}\right) .
\end{aligned}
$$

Since $\left\{V_{s}\right\}_{-\infty<s<+\infty}$ is ergodic, we have

$$
\hat{f}_{t}(y)=\left\{\begin{array}{lll}
c_{t} \cdot \exp i \xi_{t}(y) & \text { if } & t \in \sigma\left(\left\{U_{s}\right\}\right) \cap \sigma\left(\left\{V_{s}\right\}\right) \\
0 & \text { if } & t \in \sigma\left(\left\{U_{s}\right\}\right) \backslash \sigma\left(\left\{V_{s}\right\}\right)
\end{array}\right.
$$

where $c_{t}$ is a constant. Hence we have

$$
\hat{f}_{t}(y)=0, \quad \text { a.e. } y \text {, }
$$

for any $t \in \sigma\left(\left\{U_{s}\right\}\right)$ from the assumption on $f$.

Next consider the case (2) and take a measurable bounded function $\xi(x)$ which is orthogonal to every $\exp i \xi_{t}(x), t \in \sigma\left(\left\{U_{s}\right\}\right)$. Defining $\hat{f}_{\xi}(y)$ $=\langle f(\cdot, y), \xi\rangle_{L^{2}\left(\mu_{X}\right)}$, we will see $\hat{f}_{\xi}(y)=0$, a.e. $y$. Indeed

$$
\begin{aligned}
\hat{f}_{\xi}\left(V_{s} y\right) & =\left\langle f\left(\cdot, V_{s} y\right), \xi\right\rangle_{L^{2}\left(\mu_{X}\right)} \\
& =\left\langle f\left(U_{s}, y\right), \xi\right\rangle_{L^{2}\left(\mu_{X}\right)} \\
& =\left\langle f(\cdot, y), \xi\left(U_{-s^{-}}\right)\right\rangle_{L^{2}\left(\mu_{X}\right)}
\end{aligned}
$$


From Stone's spectral decomposition theorem,

$$
\xi\left(U_{s} \cdot\right)=\int_{-\infty}^{\infty} \exp i s \lambda \cdot \mathrm{d} E(\lambda) \xi
$$

Then

$$
\hat{f}_{\xi}\left(V_{s} y\right)=\int_{-\infty}^{\infty} \exp i s \lambda \cdot \mathrm{d}<f(\cdot, y), E(\lambda) \xi(\cdot)>_{L^{2}\left(\mu_{X}\right)} .
$$

We put $\mathrm{d} F(\lambda)=\mathrm{d}<f(\cdot, y), E(\lambda) \xi(\cdot)>_{L^{2}\left(\mu_{X}\right)}$. This measure is non-atomic since $\xi(\cdot)$ is orthogonal to all eigenfunctions of $\left\{U_{s}\right\}_{-\infty<s<+\infty}$. Therefore

$$
\frac{1}{s} \int_{0}^{s}\left|\hat{f}_{\xi}\left(V_{s} y\right)\right|^{2} \mathrm{~d} s=\iint \frac{\exp i s\left(\lambda^{\prime}-\lambda\right)}{s\left(\lambda^{\prime}-\lambda\right)} \mathrm{d} F(\lambda) \mathrm{d} F\left(\lambda^{\prime}\right), \quad \text { a.e. } y .
$$

The right tcrm converges to 0 as $s \rightarrow \infty$ by Lebesgue's convergence theorem. Since $\left\{V_{s}\right\}_{-\infty<s<+\infty}$ is ergodic finite measure preserving, from Birkhoff's pointwise ergodic theorem we have $\hat{f}_{\xi}(y)=0$, a.e. $y$.

Thus for almost all $y, f(x, y)$ is orthogonal to any $\mathfrak{B}_{X}$-measurable bounded function and so $f(x, y)=0$, a.e. $(x, y)$. This means that the all $\left\{U_{s} \times V_{-s}\right\}_{-\infty<s<+\infty}$-invariant square integrable functions are generated by $\left\{\exp i \xi_{t}(x) \exp i \eta_{t}(y) ; t \in \sigma\left(\left\{U_{s}\right\}\right) \cap \sigma\left(\left\{V_{s}\right\}\right)\right\}$ and that $\left\{(U, V)_{s}\right\}_{-\infty<s<+\infty}$ has the pure point spectrum $\sigma\left(\left\{U_{s}\right\}\right) \cap \sigma\left(\left\{V_{s}\right\}\right)$.

Q.E.D.

Corollary 1 (cf. [1]). Let $G_{\lambda}$ be of type $\operatorname{III}_{\lambda}(0<\lambda<1)$ and $G$ be a cyclic ergodic non-singular transformation group. Then

(1) $G$ is of type $\mathrm{III}_{\lambda^{1 / k}}$ for some integer $k$ or is of type $\mathrm{III}_{1}$ if $G_{\lambda} \times G$ is weakly equivalent with $G$, and

(2) $G_{\lambda} \times G$ is of type $\mathrm{III}_{\lambda}$ if and only if $\frac{2 \pi}{\log \lambda} \in \mathrm{T}(G)$.

Proof. The proof is clear from Theorem 6.

Corollary 2 ([6]). Let $U$ and $V$ be ergodic finite measure preserving transformations of $\left(X, \mathfrak{B}_{X}, \mu_{X}\right)$ and $\left(Y, \mathfrak{B}_{Y}, \mu_{Y}\right)$ respectively and let $G$ be an ergodic non-singular transformation group of type $\operatorname{III}_{\lambda}(0<\lambda<1)$. Then $G_{U} \times G_{V}$ is of type $\mathrm{III}_{\lambda}$ if and only if $U \times V^{-1}$ is ergodic, where $G_{U}$ is the ergodic non-singular transformation group whose definition is given in Section 2. 
Proof. We have $\mathrm{T}\left(G_{U}\right)=\frac{2 \pi}{\log \lambda} \sigma_{U}$ and $\mathrm{T}\left(G_{V}\right)=\frac{2 \pi}{\log \lambda} \sigma_{V}$, where $\sigma_{U}$ is the point spectrum set of $U$, that is the set of all $t \in \mathrm{R}$ such that there exists an $L^{2}\left(\mu_{X}\right)$-function $\xi$ satisfying $\xi(U x)=\exp 2 \pi i t . \xi(x)$, a.e. $x$. By Theorem 6, $G_{U} \times G_{V}$ is of type $\mathrm{III}_{\lambda}$ if and only if $\sigma_{U} \cap \sigma_{V}=Z$. By Lemma $1, \sigma_{U} \cap \sigma_{V}=Z$ if and only if $U \times V^{-1}$ is ergodic.

\section{§4. A Remark on the Existence of an Invariant Measure}

In [6] we showed the existence of an invariant measure under the condition that the $\mathrm{T}$-set $\mathrm{T}(G)=\mathbf{R}$. It is a measure-theoretical proof of the Connes' theorem for von Neumann algebras ([3]).

Here we give another proof of this theorem by using the associated flow.

Lemma 2. Let $\left\{U_{s}\right\}_{-\infty<s<+\infty}$ be a measurable non-singular flow of a probability space $(X, \mathfrak{B}, \mu)$. Then

$$
\int\left|f\left(U_{s} x\right) \sqrt{\frac{\mathrm{d} \mu U_{s}}{\mathrm{~d} \mu}(x)}-f\left(U_{s_{0}} x\right) \sqrt{\frac{\mathrm{d} \mu U_{s_{0}}}{\mathrm{~d} \mu}(x)}\right|^{2} \mathrm{~d} \mu(x) \rightarrow 0
$$

as $s \rightarrow s_{0}$ for $f \in L^{2}(X, \mathfrak{B}, \mu)$.

$$
\int\left|h\left(U_{s} x\right)-h\left(U_{s_{0}} x\right)\right|^{2} \mathrm{~d} \mu(x) \rightarrow 0
$$

as $s \rightarrow s_{0}$ for $h \in L^{\infty}(X, \mathfrak{B}, \mu)$.

Proof. (1) We put $\alpha(s, x)=\frac{\mathrm{d} \mu U_{s}}{\mathrm{~d} \mu}(x)$ and for $N>0, \alpha_{N}(s, x)=\alpha(s, x)$ if $0<\alpha(s, x) \leqq N$ and $\alpha_{N}(s, x)=N$ if $\alpha(s, x)>N$ which are $(s, x)$-measurable functions. We denote by $\mathbf{U}_{s}$ the unitary operator $\left(\mathbf{U}_{s} f\right)(x)=f\left(U_{s} x\right) \sqrt{\frac{\mathrm{d} \mu U_{s}}{\mathrm{~d} \mu}(x)}$. For $|s|<1$ and $f \in L^{\infty}(X, \mathfrak{B}, \mu)$ with $|f(x)|<C$,

$$
\begin{aligned}
& \left\|\mathbf{U}_{s} f(x)-f(x)\right\|_{L^{2}(\mu)}=\int_{0}^{1}\left\|\mathbf{U}_{s+u} f(x)-\mathbf{U}_{u} f(x)\right\|_{L^{2}(\mu)} \mathrm{d} u \\
& \leqq 2 C \int_{-1}^{2}\left\|\sqrt{\alpha(u, x)}-\sqrt{\alpha_{N}(u, x)}\right\|_{L^{2}(\mu)} \mathrm{d} u \\
& \quad+\int_{0}^{1}\left\|f\left(U_{s+u}(x)\right) \sqrt{\alpha_{N}(s+u, x)}-f\left(U_{u}(x)\right) \sqrt{\alpha_{N}(u, x)}\right\|_{L^{2}(\mu)} \mathrm{d} u
\end{aligned}
$$


For any $\varepsilon>0$ there exists $N$ such that

$$
\int_{-1}^{2}\left\|\sqrt{\alpha(u, x)}-\sqrt{\alpha_{N}(u, x)}\right\|_{L^{2}(\mu)} \mathrm{d} u<\varepsilon
$$

From Fubini's theorem

$$
\begin{aligned}
& \int_{0}^{1}\left\|f\left(U_{s+u}(x)\right) \sqrt{\alpha_{N}(s+u, x)}-f\left(U_{u}(x)\right) \sqrt{\alpha_{N}(u, x)}\right\|_{L^{2}(\mu)} \mathrm{d} u \\
& \quad \leqq\left\{\int \mathrm{d} \mu(x) \int_{0}^{1}\left|f\left(U_{s+u}(x)\right) \sqrt{\alpha_{N}(s+u, x)}-f\left(U_{u}(x)\right) \sqrt{\alpha_{N}(u, x)}\right|^{2} \mathrm{~d} u\right\}^{\frac{1}{2}} .
\end{aligned}
$$

From the Riemann-Lebesgue theorem

$$
\int_{0}^{1}\left|f\left(U_{s+u}(x)\right) \sqrt{\alpha_{N}(s+u, x)}-f\left(U_{u}(x)\right) \sqrt{\alpha_{N}(u, x)}\right|^{2} \mathrm{~d} u \rightarrow 0
$$

as $s \rightarrow 0$, a.e. $x$. Therefore from Lebesgue's convergence theorem

$$
\int \mathrm{d} \mu(x) \int_{0}^{1}\left|f\left(U_{s+u}(x)\right) \sqrt{\alpha_{N}(s+u, x)}-f\left(U_{u}(x)\right) \sqrt{\alpha_{N}(u, x)}\right|^{2} \mathrm{~d} u \rightarrow 0
$$

as $s \rightarrow 0$. Since $\mathbf{U}_{s}$ is a unitary operator,

$$
\left\|\mathbf{U}_{s} f-f\right\|_{L^{2}(\mu)} \rightarrow 0 \quad \text { as } \quad s \rightarrow 0
$$

for any $f \in L^{2}(X, \mathfrak{B}, \mu)$.

(2) For $h \in L^{\infty}(X, \mathfrak{B}, \mu)$ with $|h(x)|<C$,

$$
\begin{aligned}
& \left\|h\left(U_{s+s_{0}} x\right)-h\left(U_{s_{0}} x\right)\right\|_{L^{2}(\mu)} \\
& \quad \leqq\left\|h\left(U_{s+s_{0}} x\right)-\mathbf{U}_{s} h\left(U_{s_{0}} x\right)\right\|_{L^{2}(\mu)}+\left\|\mathbf{U}_{s} h\left(U_{s_{0}} x\right)-h\left(U_{s_{0}} x\right)\right\|_{L^{2}(\mu)} \\
& \quad \leqq C\left\|1-\mathbf{U}_{s} \cdot 1\right\|_{L^{2}(\mu)}+\left\|\mathbf{U}_{s} h\left(U_{s_{0}} x\right)-h\left(U_{s_{0}} x\right)\right\|_{L^{2}(\mu)} \cdot
\end{aligned}
$$

From (1),

$$
\left\|h\left(U_{s} x\right)-h\left(U_{s_{0}} x\right)\right\|_{L^{2}(\mu)} \rightarrow 0 \quad \text { as } \quad s \rightarrow s_{0} \text { 。 }
$$

Q.E.D.

Proposition 1. Let $\left\{U_{s}\right\}_{-\infty<s<+\infty}$ be a measurable ergodic nonsingular flow of a Lebesgue measure space $(X, \mathfrak{B}, \mu)$. If $\sigma\left(\left\{U_{s}\right\}\right)=\mathbf{R}$, 
then $\left\{U_{s}\right\}_{-\infty<s<+\infty}$ is metrically isomorphic with the translation on $\mathbf{R}$.

Proof. The proof is similar to the one of Theorem 3 of [6] and we use the well-known lemmas quoted in [6]. First we show that there exists a $(t, x)$-measurable function $\exp i \xi(t, x)$ such that for $-\infty<s<+\infty$

$$
\exp i \xi\left(t, U_{s} x\right)=\exp i s t \cdot \exp i \xi(t, x), \quad \text { a.e. }(t, x) .
$$

We may assume that $\mu$ is a probability measure. Let $\Gamma$ be the set of all complex valued measurable functions with absolute value 1 on $(X$, $\mathfrak{B}, \mu)$ and $\Gamma_{0}$ be the set of constant functions of $\Gamma . \quad \Gamma$ is a complete separable metric space under the relative $L^{2}(\mu)$-topology on $\Gamma$. Under the multiplication, $\Gamma$ is a topological group with respect to this topology and $\Gamma_{0}$ is its closed subgroup. From Lemma 5 ([6]) there exists a Borel subset $B$ of $\Gamma$ that intersects each coset of the quotient space $\Gamma / \Gamma_{0}$ in exactly one point. We denote by $\pi$ the canonical mapping from $\Gamma$ onto $\Gamma / \Gamma_{0}$ and denote by $\left.\pi\right|_{B}$ the restriction to $B$. For each $-\infty$ $<s<+\infty$ and $E \in \mathfrak{B}$ we denote by $\tau_{s, E}$ a function

$$
\exp i \xi(\cdot) \Gamma_{0} \rightarrow \int_{E} \exp i\left\{\xi\left(U_{s} x\right)-\xi(x)\right\} \sqrt{\frac{\mathrm{d} \mu U_{s}}{\mathrm{~d} \mu}(x)} \mathrm{d} \mu(x)
$$

defined on $\Gamma / \Gamma_{0}$. Since

$$
\begin{aligned}
& \left|\int_{E}\left\{\exp i\left\{\xi^{\prime}\left(U_{s} x\right)-\xi^{\prime}(x)\right\}-\exp i\left\{\xi\left(U_{s} x\right)-\xi(x)\right\}\right\} \sqrt{\frac{\mathrm{d} \mu U_{s}}{\mathrm{~d} \mu}(x)} \mathrm{d} \mu(x)\right| \\
& \quad \leqq 2\left(\int\left|\exp i \xi^{\prime}(x)-\exp i \xi(x)\right|^{2} \mathrm{~d} \mu(x)\right)^{\frac{1}{2}},
\end{aligned}
$$

the function $\left.\tau_{s, E^{\circ}} \pi\right|_{B}$ defined on $B$ is continuous under the relative $L^{2}(\mu)$ topology on $B$. Let $\mathbb{E}$ be the smallest $\sigma$-algebra of $\Gamma / \Gamma_{0}$ such that every function $\tau_{s, E},-\infty<s<+\infty, E \in \mathfrak{B}$, is measurable. We prove that $\mathbb{E}$ has a countably separating base. It is enough to show that for a countably separating base $\left\{E_{n}\right\}_{n \geqq 1}$ of $\mathfrak{B}$ and a countable dense set $K$ of $\mathrm{R}, \mathfrak{E}$ is generated by $\tau_{s, E_{n}}, s \in K, n \geqq 1$. From Lemma 2, for $s \in \mathrm{R}$ and $E \in \mathfrak{B}$ there exist $s_{n} \in K$ and $E_{m_{n}}$ such that

$$
\tau_{s_{n}, E_{m_{n}}}\left(\exp i \xi(\cdot) \Gamma_{0}\right) \rightarrow \tau_{s, E}\left(\exp i \xi(\cdot) \Gamma_{0}\right), \quad \text { as } \quad n \rightarrow \infty
$$

Since $\left.\tau_{s, E} \circ \pi\right|_{B},-\infty<s<+\infty, E \in \mathfrak{B}$ is continuous, $\left.\pi\right|_{B}$ is measurable 
under the $\sigma$-algebra generated by the relative $L^{2}(\mu)$-topology of $B$ and the $\sigma$-algebra $\mathfrak{E}$. From Lemma 7 ([6]) the inverse mapping $\left.\pi\right|_{B} ^{-1}$ is also measurable. For each $t \in \mathbb{R}$, let $\Gamma_{t}$ be the set of all $\mathfrak{B}$-measurable solution $\exp i \xi(\cdot)$ of the equation, for $-\infty<s<+\infty$, exp $i \xi\left(U_{s} x\right)=\exp$ its. $\exp i \xi(x)$, a.e. $x$. Then $\Gamma_{t}$ is a coset in $\Gamma / \Gamma_{0}$. By $\alpha$ we denote a mapping $t \rightarrow \Gamma_{t}$ from $\mathbf{R}$ into $\Gamma / \Gamma_{0}$. Since the function

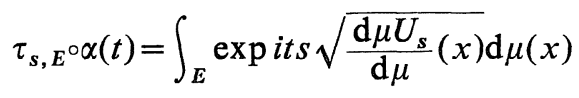

is $t$-continuous for each $-\infty<s<+\infty, E \in \mathfrak{B}$, the mapping $\alpha$ is measurable. For each $E \in \mathfrak{B}$ we denote by $\gamma_{E}$ the function $\exp i \xi(\cdot) \rightarrow$ $\int_{E} \exp i \xi(x) \mathrm{d} \mu(x)$ defined on $B$. The function $\gamma_{E}$ is continuous under the $L^{2}(\mu)$-topology. Since $\alpha,\left.\pi\right|_{B} ^{-1}$ and $\gamma_{E}$ are all measurable, the function $\left.\gamma_{E} \circ \pi\right|_{B} ^{-1} \circ \alpha(t)=\int_{E} \exp i \xi_{t}(x) \mathrm{d} \mu(x)$ is $t$-measurable for each $E \in \mathfrak{B}$, where $\exp i \xi_{t}(\cdot)=\left.\pi\right|_{B} ^{-1} \Gamma_{t}$. From Lemma 3.1 of [20], there exists a $(t, x)$-measurable function $\exp i \xi(t, x)$ such that for almost all $t, \exp i \xi(t, x)=\exp i \xi_{t}(x)$ holds except a $x$-null set. Then $\exp i \xi(t, x)$ satisfies the equation (*). For a fixed $x_{0} \in X$ the function

$$
\exp i \bar{\xi}(t, x)=\exp i\left\{\xi(t, x)-\xi\left(t, x_{0}\right)\right\}
$$

belongs to the coset $\Gamma_{t}$ and satisfies for almost all $x$

$$
\exp i \bar{\xi}(t+\tau, x)=\exp i \bar{\xi}(t, x) \cdot \exp i \bar{\xi}(\tau, x), \quad \text { a.e. }(t, \tau)
$$

From Lemma 9 ([6]) there exists a real measurable function $\xi(x)$ such that for almost all $x$

$$
\exp i \bar{\xi}(t, x)=\exp i t \xi(x), \quad \text { a.e. } t
$$

Since $\exp i \bar{\xi}(t, \cdot) \in \Gamma_{t}$, for $-\infty<s<+\infty$ and for almost all $x$

$$
\exp i t \xi\left(U_{s} x\right)=\exp i t s \cdot \exp i t \xi(x), \quad \text { a.e. } t
$$

Therefore we have for $-\infty<s<+\infty$

$$
\xi\left(U_{s} x\right)=\xi(x)+s, \quad \text { a.e. } x
$$

Then the flow $\left\{U_{s}\right\}_{-\infty<s<+\infty}$ is metrically isomorphic with the transla- 
tion on $\mathrm{R}$ under the mapping $x \rightarrow \xi(x)$ from $X$ onto $\mathbf{R}$.

Q.E.D.

Remark. If $\left\{U_{s}\right\}_{-\infty<s<+\infty}$ is not necessarily ergodic, then $\left\{U_{s}\right\}_{-\infty}$ $<s<+\infty$ is metrically isomorphic with a flow $\left(x_{0}, u\right) \rightarrow\left(x_{0}, u+s\right),-\infty<s$ $<+\infty$, defined on a measure space $X_{0} \times \mathbf{R}$ under the same condition of Proposition 1.

Corollary ([6], Theorem 3). Let $G$ be a countable non-singular transformation group. If the $\mathrm{T}$-set $\mathrm{T}(G)=\mathbf{R}$, then $G$ admits an equivalent $\sigma$-finite invariant measure.

Proof. The proof follows from Theorem 2, Proposition 1 and Theorem 3.

\section{References}

[1] Araki, H. and Woods, E.J., A classification of factors, Publ. RIMS, Kyoto Univ., 4 (1968), 51-130.

[2] Arnold, L. K., On $\sigma$-finite invariant measures, $Z$. Wahrscheinlichkeitstheorie und Verw. Gebiete, 9 (1968), 85-97.

[3] Connes, A., Une classification de facteurs de type III, Ann. Sci. École. Norm. Sup., 6 (1973), 133-252.

[4] Hajian, A. B., Ito, Y. and Kakutani, S., Invariant measures and orbits of dissipative transformations, Advances in Math., 9 (1972), 52-65.

[5] Hamachi, T., Equivalent measures on product spaces, Mem. Fac. Sci. Kyushu Univ., 27 (1973), 335-341.

[6] Hamachi, T., Oka, Y. and Osikawa, M., A classification of ergodic non-singular transformation groups, Mem. Fac. Sci. Kyushu Univ., 28 (1974), 113-133.

[7] Hill, D., $\sigma$-finite invariant measures on infinite product spaces, Trans. Amer. Math. Soc., 153 (1971), 347-370.

[8] Krieger, W., On non-singular transformations of a measure space I, II, $Z$. Wahrscheinlichkeitstheorie und Verw. Gebiete, 11 (1969), 83-91, 98-119.

[9] On hyperfinite factors and non-singular transformations of a measure space, preprint.

[10] On the Araki-Woods' asymptotic ratio set and non-singular transformations of a measure space, Lecture notes in Math., 160 (1970), 158-177.

[11] On a class of hyperfinite factors that arise from null-recurrent Markov chains, J. Functional Analysis, 7 (1971), 27-42.

[12] On ergodic flows and isomorphism of factors, preprint.

[13] Moore, C. C., Invariant measures on product spaces, Fifth Berkley Symposium on Math. Stat. and Prob. (1967), 447-459.

[14] von Neumann, J., Zur operatoren Methode in der klassischen Mechanik, Ann. of Math., 33 (1932), 587-642.

[15] von Neumann, J., On rings of operators, III, Ann. of Math., 41 (1940), 94-161. 
[16] Ornstein, D. S., On invariant measures, Bull. Amer. Math. Soc., 66 (1960), 297300.

[17] Rohlin, V.A., On the fundamental ideas of a measure theory, Mat. Sb., 25 (67) (1949), 107-150=Amer. Math. Soc. Transl., 71 (1952).

[18] Takesaki, M., Tomita's theory of modular Hilbert algebras, Lecture notes in Math., 128 (1970).

[19] Duality for crossed products and the structure of von Neumann algebras of type III, Acta Math., 131 (1973), 249-310.

[20] Mackey., G. W. A theorem of Stone and von-Neumann, Duke Math. J., 16 (1949), 313-326. 